Reprod. Nutr. Dévelop., 1987, 27 (1 B), 193-194.

\title{
Prévision de l'ingestibilité des foins chez le mouton à partir de leur teneur en parois
}

\author{
J. P. DULPHY
}

Laboratoire des Aliments,

I.N.R.A. Theix 63122 Ceyrat, France

Summary. The voluntary dry matter intake of 75 hays was measured on sheep and related to crude fiber (CB), lignocellulose (ADF) and total fiber (NDF) contents and to cellulase degradability. ADF was the best prediction criterion for pure grasses and NDF for natural grassland. However, for mixtures of grasses and grasses + lucernes, NDF was the best.

La prévision de l'ingestibilité des foins pour les ruminants est possible à partir de la teneur en cellulose brute $(C B)$ et en matières azotées totales (MAT) à condition de connaître l'espèce végétale à partir de laquelle le foin a été préparé et le numéro du cycle de végétation (Andrieu et al., 1981). Le problème des mélanges de graminées, et surtout de luzernes et de graminées, existe donc. Or on sait depuis longtemps (Crampton et al., 1960) que c'est la masse des parois et surtout sa vitesse de dégradation qui limitent les quantités de fourrages ingérées et un critère de prévision relativement efficace semble bien être la teneur en parois totales (Van Soest, 1965). Nous avons donc comparé à la teneur en CB celle en parois totales (NDF), ainsi que celle en lignocellulose (ADF) et la digestibilité par l'association pepsine-cellulase.

Matériel et méthodes. L'ingestibilité de 75 foins (tabl. 1) a été mesurée selon le procédé habituellement utilisé au laboratoire : 6 béliers castrés de $60 \mathrm{~kg}$ environ pour chaque fourrage, 2 semaines d'adaptation, 1 semaine de mesure, $10 \%$ de refus. Sur ces foins nous avons mesuré les teneurs en MAT, en CB, en parois (NDF et ADF de Goering et Van Soest, 1970, non corrigés pour l'azote et les cendres) ainsi que leur digestibilité (dcel) par la méthode de Aufrère (1982).

Résultats et discussion. Les ingestibilités et les caractéristiques chimiques moyennes des foins étudiés sont portées dans le tableau 1.

TABL. 1. - Caractéristiques des foins étudiés.

\begin{tabular}{|c|c|c|c|}
\hline & $\begin{array}{l}\text { Luzernes } \\
\qquad 14\end{array}$ & $\begin{array}{c}\text { Graminées pures } \\
28\end{array}$ & $\begin{array}{c}\text { Prairies naturelles } \\
33\end{array}$ \\
\hline Ingestibilité (g MS/kg $\mathrm{P}^{0,75}$ ) & $68,0 \pm 14,3$ & $53,9 \pm 9,0$ & $61,5 \pm 8,3$ \\
\hline MAT $\mathrm{g} / \mathrm{kg}$ MS & $157 \pm 18$ & $107 \pm 38$ & $118 \pm 38$ \\
\hline $\mathrm{CB} \quad \mathrm{g} / \mathrm{kg} \mathrm{MS}$ & $338 \pm 55$ & $317 \pm 39$ & $307 \pm 36$ \\
\hline NDF $\quad \mathrm{g} / \mathrm{kg} \mathrm{MS}$ & $548 \pm 76$ & $667 \pm 67$ & $651 \pm 55$ \\
\hline ADF $\quad \mathrm{g} / \mathrm{kg} \mathrm{MS}$ & $426 \pm 65$ & $398 \pm 49$ & $394 \pm 55$ \\
\hline dcel $\mathrm{g} / \mathrm{kg} \mathrm{MS}$ & $595 \pm 64$ & $587 \pm 76$ & $593 \pm 62$ \\
\hline
\end{tabular}

II serait fastidieux de présenter toutes les équations de régression obtenues en combinant les différents critères, d'autant que certaines combinaisons sont 
sans intérêt. Nous présentons donc dans le tableau 2 les relations obtenues avec un seul critère.

TABL. 2. - Caractéristiques des droites de régression (coefficient de corrélation $r$ et écart-type résiduel).

\begin{tabular}{lcccc}
\hline & Luzernes & Graminées pures & Prairies naturelles & Ensemble \\
\hline MAT & $0,50(12,9)$ & $0,41(8,5)$ & $0,35(8,0)$ & $0,50(9,9)$ \\
CB & $0,37(13,8)$ & $0,60(7,5)$ & $0,68(6,3)$ & $0,40(10,4)$ \\
NDF & $0,22(14,5)$ & $0,70(6,7)$ & $0,69(6,2)$ & $0,63(8,9)$ \\
ADF & $0,31(14,1)$ & $0,65(6,0)$ & $0,55(7,1)$ & $0,32(10,8)$ \\
dcel & $0,38(13,8)$ & $0,67(6,9)$ & $0,66(6,4)$ & $0,50(9,8)$ \\
\hline
\end{tabular}

Les prévisions sont très imprécises pour les foins de luzerne dont les ingestibilités sont très dispersées et le nombre d'échantillons réduit. Pour les autres foins le critère ADF pour ceux de graminées pures et NDF pour les prairies naturelles permettent les meilleures prévisions. L'addition du critère MAT n'apporte pas d'amélioration significative. II en est de même pour le critère dcel.

Si on regroupe les trois familles de foins, seul le critère NDF demeure intéressant, car les droites de régression sont alors beaucoup plus proches que par rapport aux critères $C B, A D F$ et dcel. Bien que les relations soient peu précises, le critère NDF est donc le meilleur pour estimer l'ingestibilité de mélanges de graminées ou de graminées + luzernes. L'insuffisance des trois autres critères est logique car on sait que ce sont les critères de dégradabilité à court terme qui sont les meilleurs prédicteurs de l'ingestibilité (Crampton, 1960 ; Demarquilly et Chenost, 1969).

La valeur élevée des écarts-types résiduels est due en partie à l'insuffisance de standardisation des mesures d'ingestibilité qui ont été effectuées à des saisons différentes et sur des animaux d'états différents. Elle devrait être diminuée en corrigeant les ingestibilités après les avoir comparées à celles d'un fourrage standard, mais il faut alors deux lots d'animaux pour obtenir l'ingestibilité d'un fourrage. Enfin l'existence de mécanismes de régulation des quantités ingérées au niveau sensoriel (goût-odeur) et métabolique empêcheront toujours d'avoir des équations de prévision aussi précises à partir des critères pariétaux que pour la digestibilité.

Andrieu J., Demarquilly C., Wegat-Litre E., Weiss Ph., 1981. In Prévision de la valeur nutritive des aliments des Ruminants. Ed. I.N.R.A. Publ., Route de St Cyr, 78000 Versailles. 598 pages.

Aufrère J., 1982. Ann. Zootech., 31, 111-130.

Crampton E. W., Donefer E., Lloyd L. E., 1960. J. anim. Sci., 19, 538.

Demarquilly C., Chenost M., 1969. Ann. Zootech., 18, 419-436.

Goering H. K., Van Soest P. J., 1970. In Forage fiber analyses. US Dept of Agriculture. Agr. Handbook $\mathrm{n}^{\circ} 379,20 \mathrm{p}$.

Van Soest P. J., 1965. J. anim. Sci, 24, 834. 